\title{
¿Estamos avanzando hacía una socio-ecología? Reflexiones sobre la integración de las dimensiones "humanas" en la ecología en el sur de América
}

\author{
Christopher B. Anderson ${ }^{1,2}$; J. Cristóbal Pizarro3; Rodrigo Estévez ; Alexandra \\ Sapoznikow ${ }^{5}$; Aníbal Pauchard ${ }^{6,7}$; Olga Barbosa ${ }^{7,8,9}$; Andrés Moreira-Muñoz ${ }^{10} \&$ \\ Alejandro E.J. VALENZUELA ${ }^{2,11}$
}

\begin{abstract}
1 Centro Austral de Investigaciones Científicas, CONICET. Ushuaia, Argentina. 2 Instituto de Ciencias Polares, Ambiente y Recursos Naturales, Univ. Nac. de Tierra del Fuego. Ushuaia, Argentina. 3 Department of Environment and Resource Studies, University of Waterloo, Canada. 4 Centro de Ecología Aplicada y Sustentabilidad, Depto. de Ecología, Pontificia Universidad Católica de Chile. Santiago, Chile. 5 Centro Nacional Patagónico, CONICET. Puerto Madryn, Chubut, Argentina. 6 Laboratorio de Invasiones Biológicas, Facultad de Ciencias Forestales, Universidad de Concepción. Chile. 7 Instituto de Ecología y Biodiversidad. Santiago, Chile. 8 Instituto de Ciencias Ambientales y Evolutivas, Universidad Austral de Chile. Valdivia, Chile. 9 Centro de Desarrollo Urbano Sustentable. Santiago, Chile. 10 Instituto de Geografía, Pontificia Universidad Católica de Valparaíso. Valparaíso, Chile. 11 Coordinación Patagonia Austral, Administración de Parques Nacionales. Ushuaia, Argentina.
\end{abstract}

[Palabras clave: ciencia ciudadana, ética, interdisciplinariedad, sistemas socioecológicos]

[Keywords: citizen science, ethics, interdisciplinarity, socioecological systems]

\section{INTRODUCCIÓN}

La complejidad y la velocidad de los actuales cambios ambientales a escala global presentan desafíos y oportunidades para la investigación, la gestión y la conservación de la biosfera (Millenium Ecosystem Assessment [MA] 2005). La magnitud de estos cambios derivó en la propuesta de llamar Antropoceno a nuestra época geológica (Crutzen 2002), ya que los seres humanos nos hemos convertido en la mayor fuerza de cambio planetario. Es evidente que los grandes factores promotores de este nuevo escenario (e.g., el calentamiento global, la urbanización y la globalización cultural y económica) afectan a los ecosistemas (Ellis et al. 2011), aunque también existe un reconocimiento creciente de que estos factores influyen sobre las sociedades humanas (MA 2005). Por lo tanto, se puede decir que los problemas categorizados tradicionalmente como "ambientales" (e.g., el cambio climático) poseen causas y consecuencias sociales (e.g., políticas energéticas para combustibles fósiles, impacto económico de la desertificación). De manera recíproca, los problemas "humanos" (e.g., planificación territorial y políticas de inmigración) están vinculadosa losecosistemas y a la biodiversidad (e.g., fragmentación del

Editor asociado: Diego Vázquez paisaje, introducción de especies no-nativas y homogeneización biótica).

En el contexto del Antropoceno, se hace urgente integrar las dimensiones ecológicas y las sociales del ambiente para mejorar nuestro conocimiento y tomar decisiones más efectivas (Carpenter et al. 2009). El desafío llega a ser aun más relevante en Latinoamérica, que cubriendo sólo el 10\% de la superficie del planeta alberga el 50\% de la biodiversidad terrestre (Wilson 1989) y el 15\% de la diversidad lingüística (Skutnabb-Kangas 2000). Es en Latinoamérica donde se han generado grandes conflictos socioambientales (Wagner 2010). Entonces, ¿Qué papel tiene la ecología como disciplina en Argentina y Chile con respecto a estas transformaciones? Sabemos que a nivel mundial y regional existen ejemplos de integración entre diversos campos académicos que abordan problemáticas "bioculturales" (Toledo \& Barrera 2008) y "socioecológicas" (Domptail et al. 2013) de manera interdisciplinaria. Por estas razones, y para pensarjuntos sobreesta conceptualización de lo "humano" y lo "natural" en la ecología en nuestros países, presentamos el simposio "Socio-ecología: Avanzando hacia la Integración de 'la Dimensión Humana' en el 
Estudio y Entendimiento de los Ecosistemas del Sur de Sudamérica" en la V Reunión Binacional de Ecología realizada en Chile en 2013. Las reflexiones y discusiones del simposio motivaron este artículo, que sin ser una revisión exhaustiva, intenta catalizar el debate sobre lo socioecológico en la ecología austral.

Consideramos que la interdisciplinariedad es una propiedad emergente del análisis holístico de una problemática compleja (García 2006). De esta forma quisimos superar el sesgo de la hiper-especialización individual, obstáculo para reflexiones epistemológicas y prácticas del quehacer de la ecología (Graham \& Dayton 2002). Al usar distintas perspectivas acerca de estas preguntas: a) ofrecemos una breve reseña de algunas bases epistemológicas de la dimensión humana en la ecología y revisamos las tendencias en las publicaciones socio-ecológicas a nivel mundial y regional, b) consideramos aspectos interdisciplinarios de la formación de los profesionales relacionados con la ecología y c) presentamos antecedentes de la ciencia ciudadana para ilustrar la integración de nuevos actores sociales en la investigación ecológica. Esperamos contribuir al debate sobre la postura actual y futura que pueda tener la ecología en nuestros países, y hacemos un llamado a convertirnos en agentes activos de la conceptualización y las aproximaciones de investigación y formación.

\section{LO HUMANO EN LA ECOLOGÍA}

La ecología ha experimentado vaivenes respecto a la conceptualización del ser humano dentro y fuera de la naturaleza. Estos vaivenes reflejan los cambios históricos de la relación humano-naturaleza en la cultura occidental que la originó. En el siglo $\mathrm{XV}$, y luego en el Renacimiento, la transformación de la sociedad europea desde un sistema feudal a uno burgués coincide con una separación progresiva entre lo humano y lo natural, $\mathrm{y}$ entre sujeto y objeto en la mentalidad occidental moderna (Romero 1999). En particular, en el continente americano, en los siglos XVII y XVIII, esta mentalidad influyó en la transformación del paisaje que realizaron los grupos de colonos europeos. Por ejemplo, para los puritanos en el noreste del territorio actual de los Estados Unidos (y tal vez para otras tradiciones judeo-cristianas que arribaron al continente), la naturaleza salvaje ("wilderness") fue percibida primero como el hogar del diablo y más tarde como el Edén. En ambos casos, el lugar del ser humano no estaba incluido en la naturaleza. En el primero, la naturaleza era maligna, incivilizada y diabólica; en el segundo, la presencia humana —debido al pecado original- ensuciaba a la naturaleza creada por dios, pensada como un lugar para re-crearse con la fuente divina (ver Callicott 2008).

En este entorno histórico-cultural, en el mundo anglo-parlante, la ecología se consolida a partir de la primera mitad del siglo XX con la fundación de las primeras sociedades en Gran Bretaña (1913) y Estados Unidos (1915). Como disciplina, la ecología se diferenció por estudiar las relaciones entre los componentes bióticos y abióticos del entorno, pero desde su inicio se podía apreciar una forma de pensamiento que concibió al ser humano como ajeno a este objeto de estudio. A partir de 1905, la Ecología de Comunidades, en particular la del botánico F.E. Clements, promovió a través de diversos libros y publicaciones los conceptos de sucesión vegetal (tendiente a un estado de climax) y de comunidades bióticas (como supra-organismos). Esta escuela ha sido influyente al punto de establecer en la Ecología General una concepción del mundo desde una perspectiva holística y de equilibrio similar a la visión metafísica griega de idealismo platónico y esencialismo aristotélico (Simberloff 1980). Si consideramos a esta línea de pensamiento como el primer paradigma de la disciplina, esta forma de estudiar los ecosistemas orientó la mirada de muchos ecólogos a buscar sistemas poco perturbados para entender cómo "deben ser" los ambientes naturales, pensando al ser humano como una perturbación exógena (McIntosh 1985). De la misma forma, y en su búsqueda de los principios que unifican a la ecología, el ecólogo español R. Margalef (1963) adoptó una postura que concibió la influencia humana en la naturaleza como explotación, perturbación o impedimento para que los ecosistemas alcancen su estado de "madurez".

No obstante, el pensamiento ecológico no fue del todo homogéneo (incluso en el mismo momento histórico-social) y se desarrollaron en paralelo diferentes ideas sobre la realidad. Por ejemplo, H.A. Gleason propuso una alternativa al modelo de Clements, sosteniendo que la naturaleza es un sistema dinámico, aleatorio y mecanicista. Décadas más tarde, esta idea resurgiría para impactar de forma profunda en el desarrollo 
de la ecología actual (Pickett \& Ostfeld 1995). Asimismo, varios de los fundadores de la ecología en América del Norte llamaron de manera explícita a incorporar los aspectos sociales a la disciplina. El zoólogo C.C. Adams (1940), entonces presidente de la Sociedad de Ecología de EE.UU., editó una edición especial de Ecological Monographs dedicada al vínculo entre la ecología y el bienestar humano. En su prólogo expresó que "la ecología ocupa un campo medio entre las ciencias físicas, biológicas y sociales y tiene que tratar con los valores humanos". Por otra parte, en la segunda edición del libro seminal Fundamentals of Ecology, Odum (1953) afirmó que "[se necesita] mucho estudio diligente sobre el hombre y la naturaleza (como una unidad y no [por] separado)" (traducción de los autores).

A pesar de estos llamados, el paradigma clementsiano dominó por varias décadas, y fue sólo a partir de la década del ochenta que empezó a cobrar fuerza lo que Pickett \& Ostfeld (1995) llamaron el "nuevo paradigma". Al revalorar la corriente iniciada por Gleason, estos autores desafiaron el concepto de equilibrio de la naturaleza, que tiene como corolario la naturaleza sin humanos, y propusieron que los ecosistemas son abiertos, dinámicosy relacionadosconloshumanos. Esta re-conceptualización en la ecología coincide con la creación de espacios disciplinares para la legitimación de preguntas de investigación aplicada a la sustentabilidad [véase misión de la Asociación Argentina de Ecología (AsAE 1972)]. A partir de esta aproximación, muchos ecólogos están trabajando para 1) definir sistemas socioecológicos (Haberl et al. 2006), 2) operar modelos predictivos para estudiarlos (Collins et al. 2011), 3) aplicar este conocimiento en las políticas públicas y el sector privado (Carpenter et al. 2009), y 4) desarrollar modelos de investigación y gestión del ambiente que incluyen a diversos actores sociales "in" y "ex-situ" [e.g., reservas de biosfera (Moreira-Muñoz \& Borsdorf 2014)].

En este contexto, ponemos en debate si el concepto de sistema socioecológico podría representar una forma nueva de expresar el vínculo entre humanos y no-humanos en los ecosistemas, y si podría llegar a ser una nueva manera de investigar el objeto de estudio de la ecología, haciendo explícita la integración humana. También nos preguntamos si esta conceptualización podría ofrecer un "puente" para el trabajo interdisciplinario con otros campos académicos como la Geografía, la Sociología y la Historia, entre otros. Para iniciar el diagnóstico de los alcances y usos del concepto, realizamos una búsqueda en Web of Science el 15/10/13 para publicaciones que usen las palabras clave socio-ecology, socioecology, socio-ecological, socioecological y social-ecological. Esta primera exploración fue hecha intencionalmente en inglés ya que los sistemas nacionales de ciencia, tanto en Argentina como en Chile, promueven la publicación en revistas internacionales, y también las revistas regionales suelen indexarse con resúmenes y palabras clave en español e inglés.

Al filtrar por tipo de revista encontramos que el número de publicaciones en ecología y sustentabilidad que utilizan términos socioecológicos ha aumentado en forma exponencial desde los primeros años del siglo XXI (Figura 1), mientras que en Argentina, por

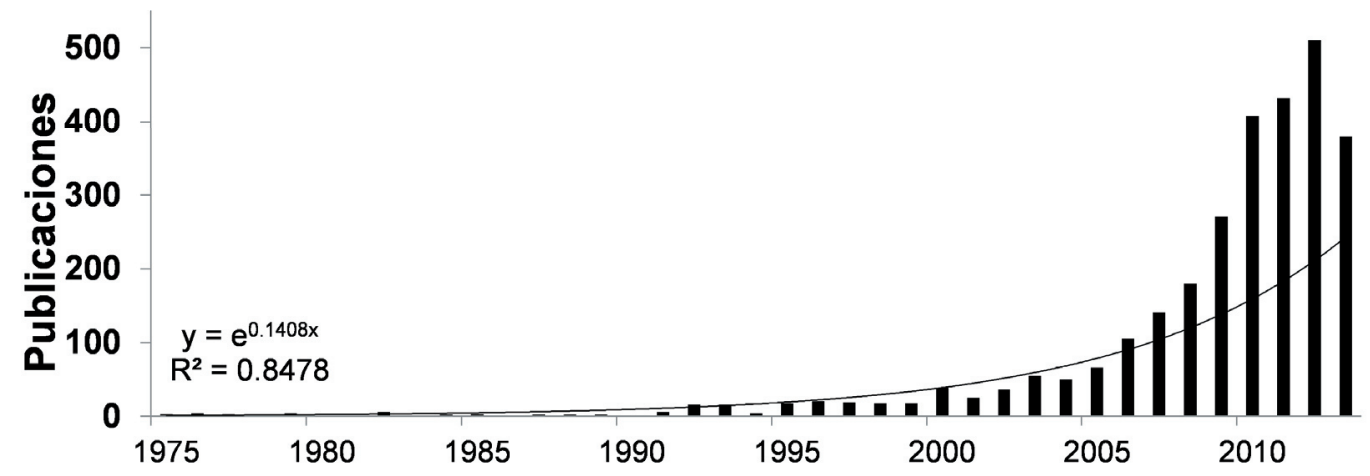

Figura 1. El uso de los términos socioecológico y social-ecológico ha crecido en forma exponencial en los artículos publicados en revistas de ecología y ciencia de la sustentabilidad en la base de datos ISI Web of Science.

Figure 1. The use of the terms socio-ecological and social-ecological has increased exponentially in ecology and sustainability science journals from the ISI Web of Science database. 
ejemplo, el crecimiento de las publicaciones en ecología en general ha aumentado de manera lineal (Anderson \& Valenzuela 2014). Estos resultados indican una tendencia ascendente en el uso de los términos socioecológicos. Sin embargo, para que estos conceptos lleguen a influir en la práctica científica, deben -además - estar asociados con una implementación activa que no sólo incluya la forma de hacer la pregunta sino también cómo abordarla a través de revistas y programas de estudio. Al respecto, a nivel mundial existen indicios de esa consolidación con nuevas revistas [e.g., Ecology \& Society; Change and Adaptation in Socio-Ecological Systems (CASES)], libros (e.g., Singh et al. 2013), programas de postgrado (e.g., maestría en Social-Ecological Resilience for Sustainable Development del Stockholm Resilience Alliance) y plataformas de Investigación Socio-Ecológica a Largo Plazo. Cada uno de estos programas y audiencias cuentan con modelos distintos de la relación ser humanonaturaleza (Maass et al. 2010). Entonces, ¿qué sucede al respecto en el sur de América?

\section{INVESTIGACIÓN ECOLÓGICA}

En Argentina y Chile, los antecedentes de la ecología se remontan a los primerosnaturalistas en la época colonial (Jaksic et al. 2012), además del conocimiento ecológico tradicional y local. No obstante, su conformación como campo académico ocurrió recién en la década del setenta. En 1972 se fundó la AsAE y en 1992 la Sociedad de Ecología de Chile (SOCECOL), que reemplazó la Sección de Ecología de la Sociedad de Biología de Chile, creada en 1978. Ambas sociedades reflejan varias nociones posteriores a la "crisis ambiental" sobre la relación ser humano-naturaleza y el auge de movimientos ambientalistas que surgieron en el mundo durante este mismo período (Estensorro Saavedra 2007). Lo cierto es que sus misiones llaman no sólo a estudiar el ambiente sino también a que los ecólogos adquieran un papel social en el desarrollo de la educación, la difusión y la solución de los problemas ambientales.

En un análisis de las investigaciones presentadas en las Reuniones Argentinas de Ecología entre 1972 y 1991, Rabinovich \& Boffi Lissin (1992) encontraron que las ramas más desarrolladas fueron la Ecología de Poblaciones, de Comunidades y la Ecofisiología. Dentro de la ecología aplicada dominó la agroecología y no hubo menciones a la ecología humana (a pesar de que era una categoría considerada en el análisis). Estos autores, además, preguntaron a los miembros de la AsAE cuáles eran las principales consecuencias ecológicas de las actividades humanas. La respuesta mayoritaria fue su capacidad de destruir hábitats, sugiriendo que en el imaginario social de los ecólogos de los años noventa, los humanos fueron conceptualizados principalmente como factor de perturbación del ambiente desde un estado "natural" previo, pese a milenios de impacto antrópico aun en el continente americano. De esta forma, hay una veta histórica que permite relacionar la ecología en nuestros países con el paradigma clementsiano, respecto al papel del ser humano en la naturaleza. De la misma forma, el Dr. Francesco Di Castri — primer catedrático de ecología en Chile- expresó: "frente a la estabilidad del ecosistema y a los juegos de equilibrio en la composición de las especies, de las edades y de los números de individuos, el hombre resulta ser el principal factor perturbador..." (Di Castri 1964).

A fin de comparar estas tendencias históricas con la producción científica actual, se revisaron todas las publicaciones en la revista Ecología Austral entre 2004 y 2014, y así determinar el tipo de investigación de cada artículo [se usaron las categorías de Rabinovich \& Boffi Lissin (1992)]. Se encontró que en la última década, la ecología de comunidades (32\%) ha superado la de poblaciones $(13 \%) \mathrm{y}$, en tercer lugar, la ecofisiología (8\%) (Figura 2A). Durante este período observamos una disminución en los artículos sobre ecología aplicada y un aumento en la ecología humana. No obstante, esta última categoría representa sólo el $4.1 \%$ del total, mientras que las investigaciones aplicadas, que suelen ser distintas formas de estudiar los impactos humanos, suman 27.5\% (Figura 2B).

\section{FORMACIÓN PROFESIONAL}

Por otra parte, la literatura académica indica que existen por lo menos tres obstáculos para formar profesionales capaces de abordar integralmentelos problemasinterdisciplinares, como los sistemas socioecológicos: i) la estructura académica/disciplinaria, ii) la implementación práctica de nuevas iniciativas, y iii) las relaciones interpersonales y de poder (Tabla 1). Los problemas estructurales en la formación se relacionan con las limitaciones 


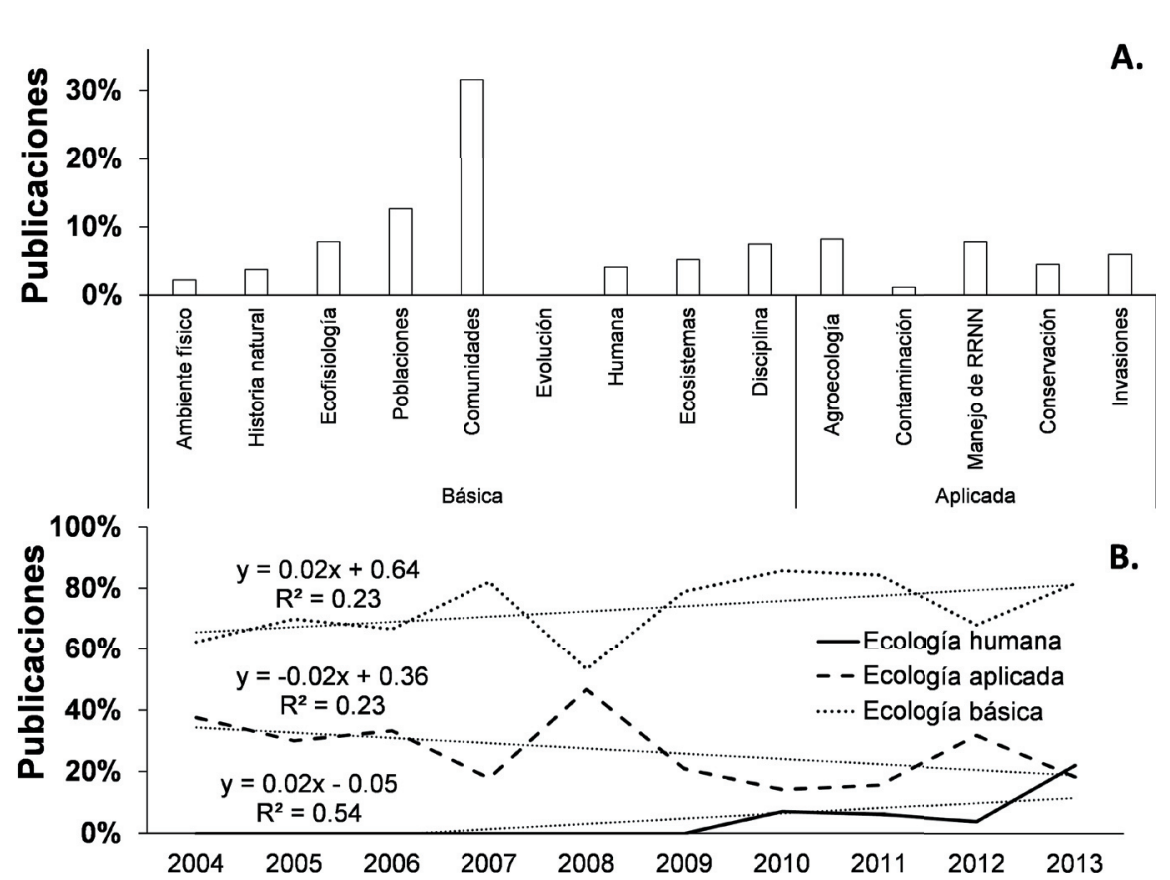

Figura 2. A)

A. Categorización de las publicaciones en la revista Ecología Austral entre 2004 y 2014. B) Tendencia temporal de publicaciones cuyo objetivo de estudio es ecología básica, aplicada

humana.

Figura 2. A) Categorization of the publications

from the journal

Ecología Austral from 2004 to 2014. B) Trend over time of the publications whose study objective is basic, applied or human.

Tabla 1. Desafíos y necesidades que moldean el perfil del profesional, investigador o técnico socioecólogo.

Table 1. Challenges and needs that influence the profile of a socioecological professional, researcher or technician.

\begin{tabular}{|c|c|c|c|}
\hline $\begin{array}{l}\text { Categoría/Desafíos } \\
\text { Estructurales }\end{array}$ & Necesidades & Capacidades a desarrollar & Referencias \\
\hline $\begin{array}{l}\text { Ofrecer una } \\
\text { aproximación } \\
\text { y contenidos } \\
\text { interdisciplinarios }\end{array}$ & $\begin{array}{l}\text { Cursos y programas de pre y } \\
\text { posgrado }\end{array}$ & $\begin{array}{l}\text { Abordaje de problemas } \\
\text { socioecológicos usando } \\
\text { aproximaciones y metodologías de } \\
\text { las ciencias sociales y la ecología }\end{array}$ & \multirow[t]{2}{*}{$\begin{array}{l}\text { Estevez et al. } 2010 \\
\text { Pretty } 2011 \\
\text { Rovere \& Tamargo } \\
2005\end{array}$} \\
\hline $\begin{array}{l}\text { Proveer dichos } \\
\text { programas con } \\
\text { financiamiento y } \\
\text { recursos básicos }\end{array}$ & $\begin{array}{l}\text { Fondos de apoyo a iniciativas } \\
\text { interdisciplinarias, Becas de } \\
\text { investigación e inserción laboral } \\
\text { Infraestructura para trabajo en } \\
\text { equipo, recursos bibliográficos }\end{array}$ & $\begin{array}{l}\text { Búsqueda de fondos concursables } \\
\text { y desarrollo y escritura de } \\
\text { propuestas de financiamiento } \\
\text { en colaboración con otros } \\
\text { investigadores }\end{array}$ & \\
\hline
\end{tabular}

Apoyar dichos programas al nivel político e institucional
Mecanismos de participación en la toma de decisiones (intra $\mathrm{e}$ interinstitucional)

Redes de colaboración interinstitucionales
Competencias políticas en organización de asociaciones y grupos de trabajo

Construcciones de redes sanas Conocimiento de gobernanza formal e informal

De implementación
Superar la brecha entre investigación Comunicación entre investigadores,
gestores, tomadores de decisiones y Desarrollo de programas de fortalecimiento a la investigación y aplicación miembros de la comunidad local
Uso de recursos no académicos
Reconocer y Mecanismos de participación resolver conflictos ciudadana
Excelentes cualidades de comunicación con distintos actores sociales y lenguajes Pietri et al. 2013

Relaciones interpersonales

\begin{tabular}{ll}
\hline $\begin{array}{l}\text { Evitar estructuras } \\
\text { piramidales y }\end{array}$ & Diversificación de la investigación y \\
las estructuras de poder
\end{tabular} piramidales y abusos de poder
Mecanismos de protección contra el abuso de poder y la discriminación
Balance entre autonomía y desarrollo de colaboraciones en la investigación.

Mares 1991

Gibson et al., 2014

Cultivar la convivencia y combatir

la discriminación en todas sus formas 
impuestas por tradiciones disciplinarias o institucionales, incluyendo la priorización de apoyo financiero o político a determinadas iniciativas (Roy et al. 2013). Por ejemplo, en los años noventa en Chile, los programas de grado y postgrado no abarcaban de manera explícita los aspectos sociales, a excepción de dos cursos de Ecología Humana (Grez et al. 1995), lo cual coincide con la tendencia histórica de excluir la dimensión humana en la ecología, o sólo considerarla como un factor de perturbación. Quince años después, Estévez et al. (2010) reportaron (en un estudio complementario sobre los programas chilenos de postgrado en conservación) que aún estos programas carecían de asignaturas con dimensiones sociales. Sólo recientemente se puede apreciar la consolidación incipiente de una oferta académica integral, con temáticas sociales. Por ejemplo, en Chile, el Doctorado en Biología y Ecología Aplicada de la U. de La Serena ofrece una asignatura titulada "Socioecología y Biología de la Conservación". No obstante, cursos como este siguen siendo casos aislados que, al parecer, no reflejan una visión nueva en la forma consolidada de concebir y de estudiar el sistema por parte de la ecología en general.

La implementación práctica de nuevas iniciativas constituye una barrera cuando no se logra integrar practicantes, tomadores de decisiones y otros actores sociales relevantes al desarrollo del conocimiento (Young et al. 2014). Este desafío es en particular relevante para la formación de profesionales que estudian sistemas socioecológicos, dada la desconexión entre la investigación académica y los "problemas del mundo real" (Pietri et al. 2013). Además, existen otros conflictos en el manejo y la dirección de los programas interinstitucionales que surgen por asimetrías de poder entre los investigadores, el Estado, las ONGs internacionales y las comunidades locales (Reyes-García et al. 2013).

Un caso regional que ha podido superar algunos aspectos de problemas estructurales y de poder surgió por una vía no tradicional al combinar instituciones académicas y el sector ONG. Se trata del Programa de Capacitación para Jóvenes Líderes de la Conservación Marina, que se originó en Argentina y luego se expandió a Chile y Uruguay. Este programa tiene como objetivo la capacitación en temas transdisciplinarios relacionados con problemas ambientales "reales". Varios cursos y talleres han sido creados y asistidos mayormente por personas con formación en ciencias naturales a fin de ofrecer conceptos y herramientas de investigación en ciencias sociales, ética, comunicación y educación, planificación y participación pública, gestión de conflictos e implementación de políticas públicas, entre otros. Las actividades incluyen análisis de casos, lecturas de artículos, juegos de roles, salidas de campo y encuentros con diferentes actores sociales, lo cual permite a los participantes comprender la complejidad del sistema tanto en sus aspectos naturales como sociales.

Esta experiencia también intenta romper con el tercer obstáculo al trabajo interdisciplinario, que incumbe las relaciones interpersonales y que reúne asuntos éticos vinculados a las asimetrías de poder entre disciplinas (naturales vs. sociales), entre la ciencia y la sociedad (expertos vs. no expertos), o entre investigadores y estudiantes (maestro vs. discípulos). Si bien se reconoce que las asimetrías de poder son una dificultad para el desarrollo y la diversificación inter- e intradisciplinaria [ver concepto de sapismo en Mares (1991)], se las ha abordado escasamente hasta no hace mucho tiempo atrás. Se sabe que estas asimetrías influyen sobre las relaciones interpersonales (Yamada et al. 2014), involucran las cualidades de los individuos e incluyen el manejo del poder de financiamiento y de las decisiones políticoinstitucionales (Gibson et al. 2014). Estas influencias incluyen no sólo asimetrías entre docentes, investigadores y estudiantes, sino también entre los tomadores de decisiones y miembros de las comunidades locales (Anderson 2014; Barnaud \& Paassen 2013). Ante esta problemática, la Red de Jóvenes Líderes de la Conservación Marina provee un espacio en donde se prioriza el vínculo horizontal entre diferentes actores en función de una visión y objetivos de conservación compartidos, y un marco de horizontalidad y confianza mutua que promueve la asociación y el intercambio de experiencias y conocimientos entre diversos actores. De esta forma se pretende expandir los alcances de la acción en un espacio de mayor incidencia que el personal o el institucional, todos ellos síntomas de una red sana (Rovere \& Tamargo 2005). Los participantes de la red han reportado que gracias a la colaboración generada en este espacio accedieron a nuevas fuentes de financiamiento, establecieron contactos con 
instituciones y personas de difícil acceso, adquirieron una nueva mirada hacia su propia práctica y generaron proyectos que integran diversas dimensiones de análisis. Esta forma de trabajo podría ofrecer un puente frente a las barreras disciplinarias y una puerta hacia nuevas fuentes de financiamiento y acuerdos institucionales.

\section{ACTORES SOCIALES}

La integración de otros actores sociales en la investigación es un tema con connotaciones tanto prácticas y teóricas como éticas y políticas. Para esta discusión nos centraremos en dos propósitos relevantes para una aproximación integral: a) crear un vínculo recíproco entre la ciencia y la sociedad, y b) realizar investigación socialmente relevante. Para la ecología, un mejor vínculo social puede proveer tanto datos como apoyo político (van Vliet et al. 2014). A su vez, la integración con otros actores - como el sector productivoresulta no sólo en aplicaciones a procesos propios del sector sino, además, en la toma de decisiones y políticas públicas alineadas con intereses sociales.

En particular, involucrarse socialmente en la toma de datos agrega una fuente nueva de información y una manera innovadora de obtenerla bajo múltiples escalas y perspectivas; esto se denomina "ciencia ciudadana" (Dickinson et al. 2012). Por ejemplo, los ciudadanos interesados en la protección ambiental pueden colectar datos sobre especies exóticas usando las tecnologías disponibles durante sus actividades habituales. Los datos luego son validados por gestores y científicos, y sirven - entre otras cosas- para delimitar con mayor precisión el rango y los avances de una invasión biológica (Crall et al. 2011). De este modo, los ciudadanos podrían alertar sobre especies nuevas (exóticas o nativas) o impactos desconocidos. Además, se educan nuevos actores, lo que permite una concienciación social sobre los componentes del cambio global, el empoderamiento en cuanto a las acciones individuales ante el fenómeno, y la demanda de acciones políticas para incluir en las agendas gubernamentales (e.g., en temas como las especies exóticas invasoras).

A su vez, cabe señalar que esta apertura no es sólo para que los ciudadanos se eduquen en la ciencia, sino también para proveer otros ciclos recíprocos de retroalimentación como la apertura de la comunidad científica hacia la sociedad y el desarrollo de temáticas o preguntas de investigación con mayor relevancia social. En este contexto, es oportuno pensar en la creación de iniciativas que generen comunidades de "co-producción" del conocimiento tanto para valorar el conocimiento no-científico de otros actores sociales (UNESCO 2005) como para generar pautas para el co-manejo de temas ambientales (Berkes 2009).

Los esquemas de ciencia participativa, de co-producción del conocimiento y de comanejo se basan sobre supuestos filosóficos que sostienen que la realidad es múltiple y co-construida, y en modelos empiristas (e.g., teoría basada en datos) acerca de asuntos o problemáticas concretas (Berkes 2009). Tales modelos podrían ser más familiares para científicos sociales que naturales (King et al. 1994). En este sentido, se advierte al lector sobre posibles dificultades en el uso de esta práctica sólo pensando en modelos teóricos hipotético-deductivos. Este debate de fondo escapa al enfoque de este artículo, pero creemos que en él reside un dilema aun mayor.

Una forma de avanzar en esta línea es la creación de redes de observadores (Sagarin \& Pauchard 2012). En términos simples, es posible distinguir tres tipos de participantes en estas redes: científicos, gestores y ciudadanos. Los científicos son los encargados de aplicar el método científico para capturar, analizar y sintetizar la información, y ayudan a mitigar los sesgos posibles de todos los observadores. Sin embargo, también presentan sesgos disciplinarios y culturales, y pueden tener agendas propias que les dificultan "observar" una realidad más amplia. Los gestores suelen estar en un contacto más cercano con los componentes del ambiente que manejan y, por lo tanto, son observadores de primera línea de los fenómenos ecológicos. Necesitan datos específicos para resolver problemas específicos, están mucho en terreno y conocen muy bien la realidad local. No obstante, sus observaciones y conclusiones pueden estar ligadas a sus convicciones "a priori" del sistema y es posible que carezcan de una perspectiva a largo plazo o un contexto más global. A su vez, los ciudadanos están en todos lados y pueden capturar datos interesantes. Sin embargo, tienen poca preparación teórica, lo que limita su capacidad de análisis e integración y puede sesgar sus observaciones 
en el "sentido común"; no obstante, también su participación integra otros saberes al diálogo con la ciencia, lo cual es necesario para generar un planeta justo y sustentable (Leff 2010).

En Argentina y Chile existen varios ejemplos de ciudadanos involucrándose en la observación ambiental (e.g., Red de Observadores de Aves y Vida Silvestre de Chile, 365 Birding Challenge y Registros Ecológicos de la Comunidad); sin emabrgo, en general se ve una escasa integración entre la investigación académica y los nuevos actores sociales, y hay un amplio espacio para mejorar el vínculo y aprovechar las sinergias y capacidades de cada grupo. Por ejemplo, en la localidad de El Chaltén, la Administración de Parques Nacionales ha iniciado un programa piloto de "ciencia ciudadana", utilizando información brindada por residentes para detectar la expansión del visón (Neovison vison), una nueva especie exótica invasora en el área. El inicio del programa consistió simplemente en brindar información a los distintos sectores sociales del lugar para la detección de la especie y posterior reporte a través de una sencilla plataforma en línea (Googledocs®). Las observaciones de los ciudadanos, promovidas por dicho programa, permitieron obtener una idea más acabada de la presencia y los usos de la especie exótica de los ambientes del área, información de base que se está utilizando para el desarrollo de un plan integral de manejo.

La ecología está acostumbrada a hacer inferencias con datos colectados para múltiples usos. Es usual que la información que proveniene de fuentes inesperadas (e.g., imágenes satelitales militares) sirva después para fines de conservación (e.g., la definición de la distribución de una especie en peligro). El desafío actual es agregar nuevos actores sociales en la investigación ecológica a fin de mejorar no sólo la captación de datos sino también la relevancia de las preguntas que se hacen y la integración social de la práctica científica (Sagarin \& Pauchard 2012).

\section{CONCLUSIONES}

Desde comienzos del siglo XXI se ha generado una discusión importante sobre el papel social del ecólogo. También, una revisión de los índices de Ecología Austral en este mismo período muestra una participación activa en el debate y una diversificación en las aproximaciones y los temas de investigación que integran el ser humano a la naturaleza (e.g., percepciones sociales, planificación o servicios ecosistémicos). Estas tendencias coinciden con un reconocimiento de la necesidad de vincular la investigación básica con iniciativas aplicadas en la ecología, incluyendo la educación y la toma de decisiones (Lubchenco et al. 1991). Dentro de los vaivenes que ha experimentado la ecología en cuanto a cómo considerar al ser humano dentro de la naturaleza, la propuesta de usar el marco conceptual de los sistemas socioecológicos puede ser novedosa. En definitiva, el ser humano no es simplemente un factor de perturbación. Además, esta apertura epistemológica podría tener una resonancia mayor para formar ecólogos más capaces de interactuar y colaborar con otras disciplinas que tienen una larga tradición "socioecológica", como — por ejemplo- la geografía humana o la etnobotánica.

En los niveles nacional y regional, esta tendencia de integración entre lo natural y lo social se ve reflejada en las prioridades que plantean las agencias de financiamiento de la ciencia. Por ejemplo, en el Programa de Desarrollo Social y Tecnológico (CONICETArgentina) y en los Proyectos IDeA (CONICYT-Chile), los indicadores de éxito no sólo son las publicaciones científicas sino también la obtención de resultados relevantes socialmente. Para que la ecología sea importante para dichas iniciativas debemos reflexionar acerca del marco conceptual con el que consideramos a nuestra disciplina, ya que luego influye en los objetos de estudio y en las aproximaciones que usamos. El surgimiento de un nuevo paradigma "socioecológico" podría ayudar a orientar nuestra mirada a la integración de los factores humanos y naturales del ambiente. No obstante, esta posibilidad está supeditada a la capacidad de consolidar e implementar las estructuras necesarias.

Así, la formación de nuevos investigadores y profesionales con una aproximación integral a la ecología va a requerir un cambio sistemático en los planes de estudios y los programas académicos en Argentina y Chile. Lo anterior implicará reformas no sólo en el diseño de las materias por parte de los docentes-investigadores sino también en la implementación de nuevas pautas de evaluación en los sistemas de acreditación impuestos por las comisiones nacionales de evaluación y acreditación. Los cambios en la institucionalización tendrían que incluir 
líneas de financiamiento específico para dichos programas y promover el desarrollo de alternativas académicas interdisciplinarias en socioecología. Esto, además, debería incidir de manera positiva en el campo laboral de los graduados, que hoy se restringe casi exclusivamente a la academia. Finalmente, el ejemplo de la ciencia ciudadana también demuestra la oportunidad de generar nuevas plataformas de investigación con otros actores sociales no tradicionales para poder realizar mejor investigación pero también orientar las preguntas y los productos de investigación a temáticas socialmente relevantes.

Agradecimientos: La participación de los expositores en el simposio fue financiado por la ONG OSARA (www.osara.org), los Fondos Basales de CONCYT (PFB-23) e ICM (PO5-002). Se agradecen los siguientes proyectos: CBA-ECO-Link (www.ecolink.frec.vt.edu, NSF GEO 1262148); JCP-BecasChile-CONICYT; OB-proyectos FONDECYT \#11110183 y FONDAP \#1510020.

\section{REFERENCIAS}

AdAMS, CC. 1940. Introductory note on symposium "Ecology and Human Welfare." Ecol. Monographs, 10: 309-310.

ANDERSON, CB. 2014. Lecciones sobre la creación e implementación de la Reserva de la Biosfera Cabo de Hornos como plataforma de investigación de largo plazo. Pp. 250-265 en: Moreira-Muñoz, A \& A Borsdorf (eds.). Reservas de la Biosfera de Chile: Laboratorios para la Sustentabilidad. Austrian Academy of Science, Pontificia Universidad Católica de Chile, Instituto de Geografía, Santiago, serie Geolibros 17.

Anderson, CB \& AEJ Valenzuela. 2014. Do what I say, not what I do. Are we linking research and decisionmaking about invasive species in Patagonia?. Ecol. Aust., 24:193-204.

BARnAUd, C \& A PAASSEn. 2013. Equity, power games and legitimacy: dilemmas of participatory natural resource management. Ecol. Soc., 18:21.

BERKES, F. 2009. Evolution of co-management: Role of knowledge generation, bridging organizations and social learning. J. Environ. Manag., 90:1692-1702.

Callicott, JB. 2008. ¿Qué wilderness en ecosistemas de frontera? Environ. Ethics, 30:235-249.

Carpenter, SR; HA MoOneY; J Agard; D Capistrano; RS DeFries; ET AL. 2009. Science for managing ecosystem services. PNAS, 106:1305-1312.

Collins, SL; SR Carpenter; SM Swinton; DE Orenstein; DL ChILDERS ET AL. 2011. An integrated conceptual framework for long-term social-ecological research. Front. Ecol. Environ., 9:351-357.

Crall, A; G Newman; T Stohlgren; K Holfelder; J Graham; ET AL. 2011. Assessing citizen science data quality: an invasive species case study. Con. Lett., 4:433-442.

CRUtZen, PJ. 2002. Geology of mankind: the anthropocene. Nature, 415:23.

Di CASTRI, F. 1964. Posición de la ecología en la ciencia y en la sociedad actual. Anales de la Universidad de Chile, 131.
Dickinson, JL; J ShiRK; D BONTER; R BONNEY; RL CRAIN; ET AL. 2012. The current state of citizen science as a tool for ecological research and public engagement. Front. Ecol. Environ., 10:291-297.

Domptail, S; MH Easdale \& Yuerlita. 2013. Managing socio-ecological systems to achieve sustainability: a study of resilience and robustness. Environ. Pol. Governance, 23:30-45.

ElLIS, E. 2011. Anthropogenic transformation of the terrestrial biosphere. Phil. Trans. Roy. Soc. A., 369: 1010-1035.

Estenssoro SAavedra, JF. 2007. Antecedentes para una historia del debate político en torno al medio ambiente. Revista Universum, 22:92-111.

Estévez, RA; DA Sotomayor; AK Poole \& JC Pizarro. 2010. Formando una nueva generación de investigadores capaces de integrar los aspectos socio-ecológicos en conservación biológica. Rev. Chi. Hist. Nat., 83:17-25.

García, R. 2006. Sistemas Complejos: Conceptos, Método y Fundamentación Epistemológica de la Investigación Interdisciplinaria. Ed. Gedisa, Barcelona, España. Pp. 202.

Gibson, C; KE Medeiros; V Giorgini; JT Mecca, LD DEVENPORT; ET AL. 2014. A qualitative analysis of power differentials in ethical situations in academia. Ethics Behav., 24:311-25.

Graham, MH \& PK Dayton. 2002. On the evolution of ecological ideas: paradigms and scientific progress. Ecol., 83:1481-1489.

GreZ, AA; FM JAKSIC; CA Moreno \& I Serey. 1995. La enseñanza de la Ecología en Chile a niveles de pregrado y postgrado. Rev. Chi. Hist. Nat., 68:139-152.

HABERL, H; V WiniwATER; K ANDERSSON; RU AYres; C BOONE; ET AL. 2006. From LTER to LTSER. Ecol. Soc., 11(2):13. www.ecologyandsociety.org/vol11/ass2/art13/

JAKSIC, FM; P CAMUS \& SA CASTRO. 2012. Ecología y Ciencias Naturales: Historia del Conocimiento del Patrimonio Biológico de Chile. DIBAM. Santiago. Pp. 228.

King, G; R Keohane \& S Verba. 1994. Designing Social Inquiry. 1st ed. Princeton University Press, New Jersey.

KuHN, TS. 1962. The Structure of Scientific Revolutions. Univ. of Chicago Press, Chicago. Pp.264

LefF, E. 2010. Imaginarios sociales y sustentabilidad. Cultura y Representaciones Sociales, 5:42-121.

Lubchenco, J; AM Olson; LB Brubaker; SR Carpenter; MM Holland; ET AL. 1991. The sustainable biosphere initiative: an ecological research agenda. Ecology, 72: 371-412.

MaAss, M; R Díaz-Delgado; P Balvanera; A Castillo \& A MARTíneZ-YrízAR. 2010. Redes de Investigación Ecológica y Socio-Ecológica a Largo Plazo (LTER y LTSER) en Iberoamérica. Rev. Chi. Hist. Nat., 83:171-184.

MargaleF, R. 1963. On certain unifying principles in ecology. The American Naturalist, 97:357-374.

Mares, MA. 1991. How scientists can impede the development of their discipline. Pp. 57-75 en: Mares, MA \& DJ Schmidly. Latin American Mammalogy: History, Biodiversity and Conservation. University of Oklahoma Press. Pp. 468.

McIntosh, RP. 1985. The Background of Ecology: Concept and Theory. Cambridge.

Millennium Ecosystem Assessment (MA). 2005. Ecosystems 
and Human Well-Being. Oct. 9 2010. www.maweb.org.

MoreirA-Muñoz, A \& A Borsdorf (eds.). 2014. Reservas de la Biosfera de Chile: Laboratorios para la Sustentabilidad. Academia de Ciencias de Austria, Pontificia Universidad Católica de Chile, Instituto de Geografía, Santiago, serie Geolibros 17. Pp. 321.

Odum, EP. 1953. Fundamentals of Ecology $2^{\circ}$ Edition. Ed. W.B. Saunders, Philadelphia. Pp. 546.

PicketT, STA \& RS OstFeld. 1995. The shifting paradigm in ecology. Pp. 261-278 en: KNIGHT, RL \& SF Bates (eds.). A New Century for Natural Resources Management. Island Press, Washington, D.C.

Pietri, DM; GG Gurney; N Benitez-Vina, A KuKLoK; SM MAXWELL; ET AL. 2013. Practical recommendations to help students bridge the research-implementation gap and promote conservation. Conserv. Biol., 27:958-67.

RabinOvich, JE \& LD Boffi Lissin. 1992. La Ecología en la República Argentina. Ecol. Aust., 2:109-122.

Reyes-García, V; I Ruíz-Mallen; L Porter-Bolland; EGariaFRAPOLLI; EA ElLIS; ET AL. 2013. Local understandings of conservation in southeastern Mexico and their implications for community-based conservation as an alternative paradigm. Conserv. Biol., 27:856-65.

Romero, JL. 1999. Estudio de la Mentalidad Burguesa. Ed. Alianza, Buenos Aires. Pp. 170.

Rovere, M \& MDC TAMARgo. 2005. Redes y Coaliciones o Cómo Ampliar el Espacio de lo Posible. Colección Gestión Social. Universidad de San Andrés. Pp.12

Roy, ED; AT MorZILLO; F SEIJO; SMW REDdY; JM RHEMtUlLA; ET AL. 2013. The elusive pursuit of interdisciplinarity at the human-environment interface. BioScience, 63:74553.
SAgarin, R \& A PAuchard. 2012. Observation and Ecology: Broadening the Scope of Science to Understand a Complex World. Island Press, Washington, DC. Pp 213

SIMBERLOFF, D. 1980. A succession of paradigms in ecology: essentialism to materialism and probabilism. Synthese, 43:3-39.

Singh, SJ; H Haberl; M Chertow; M MirTl \& M SChMid. 2006. Long-term Socio-Ecological Research. Dordrecht, Springer. Pp. 588.

Skutnabb-Kangas, T. 2000. Linguistic Genocide in Education - or Worldwide Diversity and Human Rights? Mahwah, New Jersey \& London: Lawrence Erlbaum Associates. Pp 818

Toledo, VM \& N Barrera-Bassols. 2008. La Memoria Biocultural: La Importancia Ecológica de las Sabidurías Tradicionales. Icaria Editoriales: Perspectivas Agroecológicas. Barcelona. pp. 232.

UNESCO, 2005. Hacia las Sociedades del Conocimiento. Ediciones UNESCO, Paris. Pp. 244.

VAN VLIET, AJH; WA BRon \& S Mulder. 2014. The how and why of societal publications for citizen science projects and scientists. Int. J. Biometeorol., 58:565-77.

WAGNER, LS. 2010. Movimientos socioambientales y evaluación de impacto ambiental. Rev. Argentina de Humanidades y Ciencias Sociales, 8:1-22.

Wilson, EO. 1989. Threats to biodiversity. Scientific American, 261:108-116.

YAMADA, S; MC CAPPADOCia \& D PePLer. 2014. Workplace bullying in graduate psychology programs: student perspectives of student-supervisor relationships. Train. Educ. Prof. Psycho., 8:58-67.

Young, JC; KA WAYLEN; S SARKKI; S Albon; I BaINBridge; ET AL. 2014. Improving the science-policy dialogue to meet the challenges of biodiversity conservation. Biodivers. 